\title{
Latest progress in tyrosine kinase inhibitors
}

\author{
Tatiana V. Pospelova ${ }^{1,2}$ and Valery A. Pospelov ${ }^{1,2}$ \\ ${ }^{1}$ Institute of Cytology, Russian Academy of Sciences, St. Petersburg, Russia \\ 2 Saint Petersburg State University, St. Petersburg, Russia \\ Correspondence to: Tatiana V. Pospelova, email: tvpgroup@mail.ru \\ Keywords: cancer, mTOR, kinases, c-Met, Bcr-Abl, senescence \\ Received: March 10, 2014 \\ Accepted: March 20, 2014 \\ Published: March 20, 2014 \\ This is an open-access article distributed under the terms of the Creative Commons Attribution License, which permits unrestricted use, \\ distribution, and reproduction in any medium, provided the original author and source are credited.
}

\section{ABSTRACT:}

\section{Here we discuss the latest progress in development of some kinase inhibitors such as inhibitors of C-MET, LIM and Bcr-Abl kinases. Importantly, many oncogenic kinases signal via the mTOR pathway, suggesting a common target for drug combinations.}

Met tyrosine kinase receptor signaling is activated by its ligand, hepatocyte growth factor/scatter factor (HGF/SF) [1, 2]. MET signaling in turn increases glycolysis, oxidative phosporylation, and tumor blood volume [3]. It was shown that HGF/SF-activated Met increased Ras activity, Erk phosphorylation and cell motility [3]. Tang et al. compared the demographic and clinical characteristics of ovarian cancer patients with MET altered patients and their response to c-Met inhibitors [4]. MET nonsynonomous nucleotide variations and amplification occurred in $7.4 \%$ and $3.5 \%$ of patients, respectively. MET variations were observed only in white women with high-grade ovarian tumors, whereas amplifications were detected in both black and white women with high-grade serous ovarian primary tumors. MET alterations have been associated with resistance to therapy [5]. Thus, patients exhibiting a MET alteration did not achieve an objective response by a c-Met inhibitor therapy [4]. Preclinical experiments have shown that the simultaneous use of two inhibitors anti-Met and antiEGFR significantly enhance the effectiveness of tumor growth inhibition [6]. C-MET amplified subpopulation of cells existed prior to anti-EGFR treatment supporting idea co-treatment of patients with Met and EGFR therapies [7]. MET amplification in colorectal carcinomas associated with resistance to cetuximab and panitumumab [8]. Taken together these results provide a strong rationale for the use of Met inhibitors to overcome drug-resistance to EGFR therapies.

Importantly, activators of c-MET are secreted by adipose-derived mesenchymal stem cells, which exacerbate oncogenic behaviour of c-Met-expressing breast cancer cells, creating an inflammatory microenvironment, thus increasing tumor growth and angiogenesis. c-Met expression is a predictive factor of cancer recurrence after autologous fat graft in post-surgery breast cancer patients [9].

Recently nuclear factor $\kappa$-B kinase (IKK) was identified a novel signaling mechanism for the regulation of mTORC2. A new inhibitor of IKK Bay 11-7082 interacts with Rictor and regulates the activity complex mTORC2. Rictor phosphorylation at T1135 was also inhibited by the IKK inhibitor Bay 11-7082 [10]. IKK regulates mTORC2 activity including phosphorylating AKT at the serine 473 and actin cytoskeleton reorganization, which is controlled by LIM kinases. The LIM kinases are promising oncotarget in several types of cancer [11-13]. The main substrate of LIM kinase is cofilin, an actindepolymerizing factor. LIMK1, a modulator of actin and microtubule dynamics, is involved in the mitotic process through inactivating phosphorylation of cofilin [14]. LIMK2 increases resistance to chemotherapeutic agents in neuroblastoma cells by regulating drug-induced cell cycle arrest [15]. A LIMK inhibitor, T56-LIMKi, inhibits LIMK2 with high specificity, while not inhibiting LIMK1 [13]. T56-LIMKi decreases phosphorylated cofilin (p-cofilin) levels and inhibits growth of glioma, schwannoma and pancreatic cancer. T56-LIMKi reduced tumor size and p-cofilin levels in the pancreatic tumors [13]. Also, thioredoxin inhibition is emerging as attractive strategy [16-18] especially employing already existing drugs such as Disulfiram for novel use [16, 19, 20].

Although tyrosine kinase inhibitors have changed therapy of chronic myeloid leukemia (CML), acquired resistance to imatinib, dasatinib or nilotinib, due to BCR-ABL1 kinase mutations, limits the therapy. Also, hematopoietic niche could protect leukemic cells from therapy [21]. Aggoune et al demonstrated that T315I mutants need either compound mutations such as E255K/ T315I or a stromal niche to escape from the toxicity of 
ponatinib [21]. Thus the hematopoietic niche plays a crucial role in conferring resistance to ponatinib, by increasing cell survival and genetic instability [21]. This is especially striking given that chronic myelogenous cells that are resistant to all kinase inhibitors are still highly sensitive to ponatinib $[22,23]$.

Inhibitors of the mTOR pathway sensitize chronic myeloid leukemia stem cells to nilotinib and restore the response of progenitors to nilotinib in the presence of stem cell factor [24-26]. Inhibitors of mTOR also sensitized tumor cells to other kinase inhibitors [27]. Noteworthy, the mTOR inhibitor rapamycin (sirolimus) also prevents cancer in animal models [28-36]. Rapamycin slows down organism aging [37-58], by preventing cellular geroconversion to senescence [59-70]. And everything that slows down aging in turn prevents cancer because cancer is an age-related disease [71, 72].

\section{ACKNOWLEDGMENTS:}

This work was funded by Program of the Russian Academy of Sciences (MCB RAS), grant from Russian Foundation for Basic Research (13-04-00552) and by the Program of Saint Petersburg State University (1.38.247.2014).

\section{REFERENCES}

1. Meng L, Shu M, Chen Y, Yang D, He Q, Zhao H, Feng Z, Liang C, Yu K. A novel lead compound CM-118: Antitumor activity and new insight into the molecular mechanism and combination therapy strategy in c-Met- and ALK-dependent cancers. Cancer Biol Ther. 2014; 15. in press

2. Cecchi F, Pajalunga D, Fowler CA, Uren A, Rabe DC, Peruzzi B, Macdonald NJ, Blackman DK, Stahl SJ, Byrd RA, Bottaro DP. Targeted disruption of heparan sulfate interaction with hepatocyte and vascular endothelial growth factors blocks normal and oncogenic signaling. Cancer Cell. 2012; 22: 250-262.

3. Natan S, Tsarfaty G, Horev J, Haklai R, Kloog Y, Tsarfaty I. Interplay between HGF/SF-Met-Ras signaling, tumor metabolism and blood flow as a potential target for breast cancer therapy. Oncoscience. 2014; 1(1): 30-38. http:// www.impactjournals.com/oncoscience/index.php?current

4. Tang C, Fontes Jardim DL, Falchook GS, Hess K, Fu S, Wheler JJ, Zinner RG, Naing A, Tsimberidou AM, De Melo Galgiato D, Westin SN, Meric-Bernstam F, Kurzrock R, Hong DS. MET nucleotide variations and amplification in advanced ovarian cancer: characteristics and outcomes with c-Met inhibitors. Oncoscience. 2014; 1(1): 5-13.

5. Kawakami H, Okamoto I, Arao T, Okamoto W, Matsumoto K, Taniguchi H, Kuwata K, Yamaguchi H, Nishio K, Nakagawa K, Yamada Y. MET amplification as a potential therapeutic target in gastric cancer. Oncotarget. 2013; 4:
9-17.

6. Wang W, Li Q, Takeuchi S, Yamada T, Koizumi H, Nakamura T, Matsumoto K, Mukaida N, Nishioka Y, Sone S, Nakagawa T, Uenaka T, Yano S. Met kinase inhibitor E7050 reverses three different mechanisms of hepatocyte growth factor-induced tyrosine kinase inhibitor resistance in EGFR mutant lung cancer. Clin Cancer Res. 2012; 18: 1663-1671.

7. Turke AB, Zejnullahu K, Wu YL, Song Y, Dias-Santagata D, Lifshits E, Toschi L, Rogers A, Mok T, Sequist L, Lindeman NI, Murphy C, Akhavanfard S, Yeap BY, Xiao $\mathrm{Y}$, Capelletti M et al. Preexistence and clonal selection of MET amplification in EGFR mutant NSCLC. Cancer Cell. 2010; 17: 77-88.

8. Bardelli A, Corso S, Bertotti A, Hobor S, Valtorta E, Siravegna G, Sartore-Bianchi A, Scala E, Cassingena A, Zecchin D, Apicella M, Migliardi G, Galimi F, Lauricella C, Zanon C, Perera T et al. Amplification of the MET receptor drives resistance to anti-EGFR therapies in colorectal cancer. Cancer Discov. 2013; 3: 658-673.

9. Eterno V, Zambelli A, Pavesi L, Villani L, Zanini V, Petrolo G, Manera S, Tuscano A, Amato A. Adipose-derived Mesenchymal Stem Cells (ASCs) may favour breast cancer recurrence via $\mathrm{HGF} / \mathrm{c}-\mathrm{Met}$ signaling. Oncotarget. 2014: 5: 613-633

10. Xu Y, Lai E, Liu J, Lin J, Yang C, Jia C, Li Y, Bai X, Li M. IKK interacts with rictor and regulates mTORC2. Cell Signal. 2013; 25: 2239-2245.

11. Prudent R, Vassal-Stermann E, Nguyen $\mathrm{CH}$, Pillet $\mathrm{C}$, Martinez A, Prunier C, Barette C, Soleilhac E, Filhol O, Beghin A, Valdameri G, Honore S, Aci-Seche S, Grierson D, Antonipillai J, Li R et al. Pharmacological inhibition of LIM kinase stabilizes microtubules and inhibits neoplastic growth. Cancer Res. 2013; 72: 4429-4439.

12. Ning H, Mitsui H, Wang CQ, Suarez-Farinas M, Gonzalez J, Shah KR, Chen J, Coats I, Felsen D, Carucci JA, Krueger JG. Identification of anaplastic lymphoma kinase as a potential therapeutic target in Basal Cell Carcinoma. Oncotarget. 2013; 4: 2237-2248.

13. Rak R, Haklai R, Elad-Tzfadia G, Wolfson HJ, Carmeli S, Kloog Y. Novel LIMK2 inhibitor blocks Panc-1 tumor growth in a mouse xenograft model. Oncoscience. 2014; 1(1): 39-48.

14. Ritchey L, Ottman R, Roumanos M, Chakrabarti R. A functional cooperativity between Aurora A kinase and LIM kinase1: implication in the mitotic process. Cell Cycle. 2012; 11: 296-309.

15. Gamell C, Schofield AV, Suryadinata R, Sarcevic B, Bernard O. LIMK2 mediates resistance to chemotherapeutic drugs in neuroblastoma cells through regulation of druginduced cell cycle arrest. PLoS One. 2013; 8: e72850.

16. Papaioannou M, Mylonas I, Kast RE, Bruning A. Disulfiram/copper causes redox-related proteotoxicity and concomitant heat shock response in ovarian cancer cells that 
is augmented by auranofin-mediated thioredoxin inhibition. Oncoscience. 2014; 1(1): 21-29.

17. Raninga PV, Di Trapani G, Tonissen KF. Cross talk between two antioxidant systems, Thioredoxin and DJ-1: consequences for cancer. Oncoscience. 2014; 1(1): 95-109.

18. Mahmood DF, Abderrazak A, El Hadri K, Simmet T, Rouis $\mathrm{M}$. The thioredoxin system as a therapeutic target in human health and disease. Antioxid Redox Signal. 2013; 19: 1266 1303.

19. Triscott J, Lee C, Hu K, Fotovati A, Berns R, Pambid M, Luk M, Kast RE, Kong E, Toyota E, Yip S, Toyota B, Dunn SE. Disulfiram, a drug widely used to control alcoholism, suppresses the self-renewal of glioblastoma and over-rides resistance to temozolomide. Oncotarget. 2012; 3: 11121123.

20. Hothi P, Martins TJ, Chen L, Deleyrolle L, Yoon JG, Reynolds B, Foltz G. High-throughput chemical screens identify disulfiram as an inhibitor of human glioblastoma stem cells. Oncotarget. 2012; 3: 1124-1136.

21. Aggoune D, Tosca L, Sorel N, Bonnet ML, Dkhissi F, Tachdjian G, Bennaceur-Griscelli A, Chomel JC, Turhan AG. Modeling the influence of stromal microenvironment in the selection of ENU-induced BCR-ABL1 mutants by tyrosine kinase inhibitors. Oncoscience. 2014; 1(1): 57-68.

22. Cassuto O, Dufies M, Jacquel A, Robert G, Ginet C, Dubois A, Hamouda A, Puissant A, Luciano F, Karsenti JM, Legros L, Cassuto JP, Lenain P, Auberger P. All tyrosine kinase inhibitor-resistant chronic myelogenous cells are highly sensitive to ponatinib. Oncotarget. 2012; 3: 1557-1565.

23. Dufies M, Cassuto O, Jacquel A, Robert G, Auberger P. Ponatinib circumvents all types of imatinib resistance in chronic myelogenous leukemia cell lines. Cell Cycle. 2013; 12: $1645-1646$.

24. Airiau K, Mahon FX, Josselin M, Jeanneteau M, Belloc F. $\mathrm{PI} 3 \mathrm{~K} / \mathrm{mTOR}$ pathway inhibitors sensitize chronic myeloid leukemia stem cells to nilotinib and restore the response of progenitors to nilotinib in the presence of stem cell factor. Cell Death Dis. 2013; 4: e827.

25. Huang ZL, Gao M, Li QY, Tao K, Xiao Q, Cao WX, Feng WL. Induction of apoptosis by directing oncogenic Bcr-Abl into the nucleus. Oncotarget. 2013; 4: 2249-2260.

26. Ciarcia R, Damiano S, Montagnaro S, Pagnini U, Ruocco A, Caparrotti G, d'Angelo D, Boffo S, Morales F, Rizzolio F, Florio S, Giordano A. Combined effects of PI3K and SRC kinase inhibitors with imatinib on intracellular calcium levels, autophagy, and apoptosis in CML-PBL cells. Cell Cycle. 2013; 12: 2839-2848.

27. Ishikawa D, Takeuchi S, Nakagawa T, Sano T, Nakade J, Nanjo S, Yamada T, Ebi H, Zhao L, Yasumoto K, Nakamura T, Matsumoto K, Kagamu H, Yoshizawa H, Yano S. mTOR inhibitors control the growth of EGFR mutant lung cancer even after acquiring resistance by HGF. PLoS One. 2013; 8: e62104.

28. Komarova EA, Antoch MP, Novototskaya LR, Chernova
OB, Paszkiewicz G, Leontieva OV, Blagosklonny MV, Gudkov AV. Rapamycin extends lifespan and delays tumorigenesis in heterozygous p53+/- mice. Aging (Albany NY). 2012; 4: 709-714.

29. Comas M, Toshkov I, Kuropatwinski KK, Chernova OB, Polinsky A, Blagosklonny MV, Gudkov AV, Antoch MP. New nanoformulation of rapamycin Rapatar extends lifespan in homozygous p53-/- mice by delaying carcinogenesis. Aging (Albany NY). 2012; 4: 715-722.

30. Donehower LA. Rapamycin as longevity enhancer and cancer preventative agent in the context of p53 deficiency. Aging (Albany NY). 2012; 4: 660-661.

31. Anisimov VN, Zabezhinski MA, Popovich IG, Piskunova TS, Semenchenko AV, Tyndyk ML, Yurova MN, Antoch MP, Blagosklonny MV. Rapamycin extends maximal lifespan in cancer-prone mice. Am J Pathol. 2010; 176: 2092-2097.

32. Popovich IG, Anisimov VN, Zabezhinski MA, Semenchenko AV, Tyndyk ML, Yurova MN, Blagosklonny MV. Lifespan extension and cancer prevention in HER-2/ neu transgenic mice treated with low intermittent doses of rapamycin. Cancer Biol Ther. 2014; 15. In press

33. Selman C, Partridge L. A double whammy for aging? Rapamycin extends lifespan and inhibits cancer in inbred female mice. Cell Cycle. 2012; 11: 17-18.

34. Liu Y, Huang Y, Wang Z, Li X, Louie A, Wei G, Mao JH. Temporal mTOR inhibition protects Fbxw7-deficient mice from radiation-induced tumor development. Aging (Albany NY). 2013; 5: 111-119.

35. Livi CB, Hardman RL, Christy BA, Dodds SG, Jones D, Williams C, Strong R, Bokov A, Javors MA, Ikeno Y, Hubbard G, Hasty P, Sharp ZD. Rapamycin extends life span of Rb1+/- mice by inhibiting neuroendocrine tumors. Aging (Albany NY). 2013; 5: 100-110.

36. Granville CA, Warfel N, Tsurutani J, Hollander MC, Robertson M, Fox SD, Veenstra TD, Issaq HJ, Linnoila RI, Dennis PA. Identification of a highly effective rapamycin schedule that markedly reduces the size, multiplicity, and phenotypic progression of tobacco carcinogen-induced murine lung tumors. Clin Cancer Res. 2007; 13: 2281-2289.

37. Blagosklonny MV. Rapamycin extends life- and health span because it slows aging. Aging (Albany NY). 2013; 5: 592598.

38. Bravo-San Pedro JM, Senovilla L. Immunostimulatory activity of lifespan-extending agents. Aging (Albany NY). 2013; 5: 793-801.

39. Fang Y, Bartke A. Prolonged rapamycin treatment led to beneficial metabolic switch. Aging (Albany NY). 2013; 5: 328-329.

40. Kennedy BK, Pennypacker JK. Drugs that modulate aging: the promising yet difficult path ahead. Transl Res. 2013.

41. Anisimov VN. Metformin: do we finally have an anti-aging drug? Cell Cycle. 2013; 12: 3483-3489.

42. Fok WC, Chen Y, Bokov A, Zhang Y, Salmon AB, 
Diaz V, Javors M, Wood WH, 3rd, Becker KG, Perez VI, Richardson A. Mice fed rapamycin have an increase in lifespan associated with major changes in the liver transcriptome. PLoS One. 2014; 9: e83988.

43. Kaeberlein M. mTOR Inhibition: From Aging to Autism and Beyond. Scientifica (Cairo). 2013; 2013: 849186.

44. Johnson SC, Martin GM, Rabinovitch PS, Kaeberlein M. Preserving youth: does rapamycin deliver? Sci Transl Med. 2013; 5: 211 fs 240.

45. Miller RA, Harrison DE, Astle CM, Fernandez E, Flurkey K, Han M, Javors MA, Li X, Nadon NL, Nelson JF, Pletcher S, Salmon AB, Sharp ZD, Van Roekel S, Winkleman L, Strong R. Rapamycin-mediated lifespan increase in mice is dose and sex dependent and metabolically distinct from dietary restriction. Aging Cell. 2013.

46. Leontieva OV, Novototskaya LR, Paszkiewicz GM, Komarova EA, Gudkov AV, Blagosklonny MV. Dysregulation of the mTOR pathway in p53-deficient mice. Cancer Biol Ther. 2013; 14. 1182 - 1188

47. Danilov A, Shaposhnikov M, Plyusnina E, Kogan V, Fedichev P, Moskalev A. Selective anticancer agents suppress aging in Drosophila. Oncotarget. 2013; 4: 15071526.

48. Khapre RV, Kondratova AA, Patel S, Dubrovsky Y, Wrobel M, Antoch MP, Kondratov RV. BMAL1-dependent regulation of the mTOR signaling pathway delays aging. Aging (Albany NY). 2014; 6: 48-57.

49. Leontieva OV, Geraldine M. Paszkiewicz GM, Blagosklonny MV. Mechanistic or mammalian target of rapamycin (mTOR) may determine robustness in young male mice at the cost of accelerated aging. Aging (Albany ny). 2012; 4: 899-916.

50. Blagosklonny MV. Prospective treatment of age-related diseases by slowing down aging. Am J Pathol. 2012; 181: 1142-1146.

51. Kolosova NG, Vitovtov AO, Muraleva NA, Akulov AE, Stefanova NA, Blagosklonny MV. Rapamycin suppresses brain aging in senescence-accelerated OXYS rats. Aging (Albany NY). 2013; 5: 474-484.

52. Longo VD, Fontana L. Intermittent supplementation with rapamycin as a dietary restriction mimetic. Aging (Albany NY). 2011; 3: 1039-1040.

53. Miller RA, Harrison DE, Astle CM, Baur JA, Boyd AR, de Cabo R, Fernandez E, Flurkey K, Javors MA, Nelson JF, Orihuela CJ, Pletcher S, Sharp ZD, Sinclair D, Starnes JW, Wilkinson JE et al. Rapamycin, but not resveratrol or simvastatin, extends life span of genetically heterogeneous mice. J Gerontol A Biol Sci Med Sci. 2011; 66: 191-201.

54. Flynn JM, O'Leary MN, Zambataro CA, Academia EC, Presley MP, Garrett BJ, Zykovich A, Mooney SD, Strong R, Rosen CJ, Kapahi P, Nelson MD, Kennedy BK, Melov $\mathrm{S}$. Late life rapamycin treatment reverses age-related heart dysfunction. Aging Cell. 2013. 12; 851-862

55. Khanna A, Kapahi P. Rapamycin: killing two birds with one stone. Aging (Albany NY). 2011; 3: 1043-1044.

56. Ye L, Widlund AL, Sims CA, Lamming DW, Guan Y, Davis JG, Sabatini DM, Harrison DE, Vang O, Baur JA. Rapamycin doses sufficient to extend lifespan do not compromise muscle mitochondrial content or endurance. Aging (Albany NY). 2013; 5: 539-550.

57. Zheng XF. Chemoprevention of age-related macular regeneration (AMD) with rapamycin. Aging (Albany NY). 2012; 4: 375-376.

58. Blagosklonny MV. TOR-centric view on insulin resistance and diabetic complications: perspective for endocrinologists and gerontologists. Cell Death Dis. 2013; 4: e964.

59. Galluzzi L, Kepp O, Kroemer G. TP53 and MTOR crosstalk to regulate cellular senescence. Aging (Albany NY). 2010; 2: 535-537.

60. Kolesnichenko M, Hong L, Liao R, Vogt PK, Sun P. Attenuation of TORC1 signaling delays replicative and oncogenic RAS-induced senescence. Cell Cycle. 2012; 11: 2391-2401.

61. Pospelova TV, Leontieva OV, Bykova TV, Zubova SG, Pospelov VA, Blagosklonny MV. Suppression of replicative senescence by rapamycin in rodent embryonic cells. Cell Cycle. 2012; 11: 2402-2407.

62. Blagosklonny MV. Cell cycle arrest is not yet senescence, which is not just cell cycle arrest: terminology for TORdriven aging. Aging (Albany NY). 2012; 4: 159-165.

63. Romanov VS, Abramova MV, Svetlikova SB, Bykova TV, Zubova SG, Aksenov ND, Fornace AJ, Jr., Pospelova TV, Pospelov VA. p21(Waf1) is required for cellular senescence but not for cell cycle arrest induced by the HDAC inhibitor sodium butyrate. Cell Cycle. 2010; 9: 3945-3955.

64. Hinojosa CA, Mgbemena V, Van Roekel S, Austad SN, Miller RA, Bose S, Orihuela CJ. Enteric-delivered rapamycin enhances resistance of aged mice to pneumococcal pneumonia through reduced cellular senescence. Exp Gerontol. 2012; 47: 958-965.

65. Halicka HD, Zhao H, Li J, Lee YS, Hsieh TC, Wu JM, Darzynkiewicz Z. Potential anti-aging agents suppress the level of constitutive mTOR- and DNA damage- signaling. Aging (Albany NY). 2012; 4: 952-965.

66. Iglesias-Bartolome R, Patel V, Cotrim A, Leelahavanichkul K, Molinolo AA, Mitchell JB, Gutkind JS. mTOR inhibition prevents epithelial stem cell senescence and protects from radiation-induced mucositis. Cell Stem Cell. 2012; 11: 401414.

67. Mercier I, Camacho J, Titchen K, Gonzales DM, Quann K, Bryant KG, Molchansky A, Milliman JN, WhitakerMenezes D, Sotgia F, Jasmin JF, Schwarting R, Pestell RG, Blagosklonny MV, Lisanti MP. Caveolin-1 and accelerated host aging in the breast tumor microenvironment: chemoprevention with rapamycin, an mTOR inhibitor and anti-aging drug. Am J Pathol. 2012; 181: 278-293.

68. Luo Y, Li L, Zou P, Wang J, Shao L, Zhou D, Liu L. Rapamycin enhances long-term hematopoietic 
reconstitution of ex vivo expanded mouse hematopoietic stem cells by inhibiting senescence. Transplantation. 2014; 97: 20-29.

69. Panganiban RA, Day RM. Inhibition of IGF-1R Prevents Ionizing Radiation-Induced Primary Endothelial Cell Senescence. PLoS One. 2013; 8: e78589.

70. Sousa-Victor P, Gutarra S, Garcia-Prat L, RodriguezUbreva J, Ortet L, Ruiz-Bonilla V, Jardi M, Ballestar E, Gonzalez S, Serrano AL, Perdiguero E, Munoz-Canoves P. Geriatric muscle stem cells switch reversible quiescence into senescence. Nature. 2014; 506: 316-321.

71. Blagosklonny MV. Rapalogs in cancer prevention: Antiaging or anticancer? Cancer Biol Ther. 2012; 13: 13491354.

72. Anisimov VN. Multifaceted aging and rapamycin. Aging (Albany NY). 2013; 5: 487. 